\title{
Correction to: Braverman and His Theory of Disequilibrium Economics
}

\author{
Mark Levin
}

\section{Correction to: \\ Chapter "Braverman and His Theory of Disequilibrium Economics" in: L. Rozonoer et al. (Eds.): Braverman Readings in Machine Learning, LNAI 11100, https://doi.org/10.1007/978-3-319-99492-5_15}

By mistake the uncorrected proof of the original chapter was published. In the updated version, numerous small corrections were made throughout the chapter.

The updated online version of this chapter can be found at https://doi.org/10.1007/978-3-319-99492-5_15 